\title{
La irrupción del teletrabajo y el eterno retorno al debate sobre la regulación del tiempo de trabajo
}

\section{The Incursion of Telework and the Constant Return of the Working Hours Regulation Debate}

Andrea Delfino

\author{
Andrea Delfino es docente e investigadora de la \\ Escuela de Ciencia Política, Facultad de Ciencia Política \\ y Relaciones Internacionales, Universidad Nacional de \\ Rosario, Argentina. \\ E-mail: andelfino@yahoo.com.ar
}

\section{resumen}

La irrupción del teletrabajo en Argentina se dio de forma abrupta a partir del establecimiento del Aislamiento Social, Preventivo y Obligatorio (ASPO) como medida sanitaria para contener la pandemia de Covid-19. Hasta ese momento, esta modalidad de trabajo no tenía una regulación específica. Finalmente, fue debatida y aprobada entre los meses de junio y agosto de 2020. Este trabajo discute tres características del teletrabajo, las cuales están íntimamente relacionadas e incluso confundidas dentro de la noción de tiempo de trabajo: la relación tiempo-espacio, los límites de la jornada de trabajo y la oposición entre tiempo de trabajo mercantil y otros tiempos necesarios para la vida. Algunos de estos elementos fueron parcialmente incorporados a la nueva regulación.

\section{summary}

The abrupt incursion of teleworking in Argentina took place after the lockdown measures taken in order to contain the spread of Covid-19 infections. Before the pandemic, telework was not specifically regulated. However, it was finally discussed and passed between the months of June and August 2020. This study discusses three characteristics of telework, which are closely related and are even confused within the notion of working time: the space and time relationship, the working hours limits, and the conflict between working time and the time necessary for other aspects of life. Some of these elements were partially incorporated into the new regulation.

\section{palabras clave}

teletrabajo / nueva institucionalidad laboral / tiempo de trabajo

\section{keywords}

telework / new labour institutionalization / working time 


\section{Introducción}

A partir del 20 de marzo de 2020, el Gobierno Nacional estableció en Argentina el Aislamiento Social, Preventivo y Obligatorio (ASPO) como medida sanitaria para contener la pandemia de Covid-19. ${ }^{1}$ Desde ese momento, casi la totalidad de la población del país entró en confinamiento y se impusieron restricciones a la circulación y a la asistencia a los lugares de trabajo ${ }^{2}$, excepto para aquellos trabajadores de las consideradas actividades esenciales. Así, una parte de la producción de bienes y servicios, tanto públicos como privados, comenzó a ser realizada de forma remota, en los hogares de trabajadores y trabajadoras y a través de diferentes tecnologías de la información y la comunicación (TICs).

La irrupción del teletrabajo se dio de forma abrupta y en ausencia de regulación específica. Entre los meses de junio y agosto de 2020 -aún en el marco de la vigencia del ASPO-, se produjo el debate parlamentario y la sanción de una ley que otorgara una nueva institucionalidad a esta modalidad de trabajo. ${ }^{3}$ El teletrabajo conlleva la particularidad de poner en discusión algunas de las características centrales del modelo temporal surgido a partir de la sociedad industrial. Presenta, así, una serie de desafíos para la nueva regulación. Este texto discute tres de esas características, las cuales están íntimamente relacionadas e incluso confundidas dentro de la noción de tiempo de trabajo: la relación tiempo-espacio, los límites a la jornada de trabajo y la oposición entre tiempo de trabajo mercantil y otros tiempos necesarios para la vida.

De este modo, partimos de la perspectiva de que el tiempo puede ser pensado como una "síntesis compleja" (Elias, 1998) y se encuentra dentro de los conflictos -visibles o invisibles- que animan la vida social y personal (Bouffartigue, 2012). En este sentido, el estudio de los tiempos de trabajo constituye una óptica privilegiada para el análisis de la desigualdad.

\section{1- Una corta perspectiva del largo plazo}

Fue durante la Edad Media que comenzó el largo proceso de transformaciones en la noción y percepción del tiempo, el cual supuso un cambio de enfoque en el tiempo de trabajo. Esto conllevó un pasaje desde el trabajo orientado hacia las tareas de la época preindustrial hacia una nueva situación en la cual el trabajo pasó a ser regulado por el tiempo de las horas. A partir de esta revolución temporal, es el ritmo estandarizado del reloj el que pasa a gobernar la rutina y el tiempo de trabajo (Thompson, 1984).

La interiorización de esa economía del tiempo útil experimentada por las personas pobres abarcó el extenso período que va entre el siglo XIV y el inicio del siglo XIX. El análisis thompsoniano asocia la actitud orientada por el tiempo de las horas con la disciplina temporal, con el ahorro del tiempo, con la clara demarcación entre trabajo y ocio, y con el más amplio proceso de mercantilización. En este contexto, sería el puritanismo, en su estrecho vínculo con el capitalismo industrial, el agente que posibilitó la conversión del hombre a los nuevos valores del tiempo, y dio lugar a la interiorización de una disciplina en relación con el tiempo. A partir de ese momento, el valor del tiempo -y no la tarea-devino en dominante. El tiem- 
po fue transformado en algo de uso generalizado y corriente, y en algo que debe ser gastado (Adam, 1995). Este proceso se habría producido independientemente de la existencia del reloj.

Thompson (1984) señala que, a partir del siglo XVIII, se intensifica la ofensiva por la consolidación de esa disciplina del tiempo con relación al trabajo, la cual forma parte desde entonces del panorama del capitalismo industrial disciplinado. El trabajo a domicilio no solo había comenzado a disminuir por razones económicas. El deseo de obtener mayores y más regulares ganancias se acompañaba del deseo de limitar el tiempo dedicado al trabajo: cuando se trabaja en la fábrica, se sabe cuándo se terminará el trabajo. El tiempo que se escapa al patrón, y cuya importancia crece a lo largo de todo el siglo, es un tiempo del que se dispone plenamente y del cual se es propietario. Trabajar fuera de la propia casa es estar plenamente en la casa propia cuando se está en ella. En este sentido, el retroceso del trabajo a domicilio responde a la reivindicación de una vida privada (Prost, 1989).

Para Giddens (1991), el invento del reloj mecánico -y su difusión a todos los miembros de la población en el siglo XVIII- fue de crucial importancia en la separación del tiempo y el espacio. El tiempo había estado conectado al espacio hasta que la uniformidad de la medida del tiempo, con el reloj, llegó a emparejarse con la uniformidad en la organización social del tiempo. Para el autor, este cambio coincidió con la expansión de la Modernidad y solo llegó a completarse hacia el siglo XX.

En este sentido, el tiempo que permite que el trabajo sea transformado en dinero es un tiempo con un valor de intercambio abstracto, descontextualizado, cuantitativo y divisible, que permite su tratamiento como una mercancía dentro del mercado de trabajo. Este tiempo mecánico y del reloj es un tiempo uniforme, invariable, divisible en infinitas unidades, al que es posible otorgarle un valor numérico. Con la mercantilización, el control del tiempo devino en un aspecto integral de la vida en sociedad, y debe ser enfatizado como un fenómeno específico de la sociedad industrial.

De allí que la esfera pública jugara un rol central en restringir la extensión de la jornada de trabajo y diera lugar al tiempo de trabajo "comprimido", que respondía a un reclamo de la clase obrera para beneficiarse realmente del tiempo suplementario de no trabajo y distanciarse de un trabajo degradado. En este sentido, la escisión y la especialización de los espacios públicos y privados, así como la delimitación de la jornada laboral, formaron parte del compromiso social construido en la esfera política entre el movimiento obrero organizado y el capital. Desde una perspectiva temporal, es posible observar cómo la relación laboral estándar estableció límites vinculados con las temporalidades en relación con dos aspectos: a) el salario que reciben los trabajadores y el esfuerzo o tiempo de trabajo requerido para la obtención de ese salario; y b) la parte del día, semana o año que se encuentra bajo el control del empleador (Rubery et al., 2005). Sin embargo, la existencia de un tiempo dominante no puede ocultar la pluralidad de los tiempos, ya que a la par de un tiempo hegemónico se despliegan otros tipos de tiempos 
construidos a partir de lógicas extra mercantiles, caracterizados por ser irregulares $\mathrm{y}$ discontinuos.

A partir de la década de 1970, las nuevas formas de organización de la producción han dado lugar a sustantivas modificaciones en las formas de organización del tiempo de trabajo, con tendencias hacia su diversificación y su heterogeneización. Las transformaciones en las relaciones laborales pueden ser vistas como una transformación de las temporalidades asociadas al trabajo remunerado. En esta transformación, cambian las formas de negociación en torno a los salarios y se produce un desdibujamiento de las fronteras entre tiempos laborales y no laborales.

Para Bouffartigue y Boutellier (2002), los factores que están en la base de estas transformaciones son numerosos y pueden ser clasificados en tres tipos: productivos, tecnológicos y socio-institucionales. En relación con los primeros, es decir, los factores productivos, es posible señalar que con el predominio de las formas de organización productivas flexibles se han diversificado las formas de contratación y de empleo, las cuales están asociadas a temporalidades cada vez más desarticuladas e inciertas.

En cuanto a los factores tecnológicos, la creciente integración de las tecnologías de la información y la comunicación en el ámbito de la producción ha sido compatible con las nuevas exigencias de flexibilidad respecto del tiempo, lugar y modo de la prestación laboral. Por un lado, ha permitido que el trabajador se desempeñe en una ubicación diferente a la empresa empleadora/cliente para la cual trabaja, lo cual favorece, así, situaciones de externalización laboral. A su vez, algunas tecnologías han generado el fenómeno de la "disponibilidad laboral permanente", lo que estimula -incluso para los asalariados típicos-el traslado de tareas a ámbitos extra-laborales, en horas y días de descanso.

Finalmente, y en relación con los factores socio-institucionales, la re-regulación de los mercados laborales posibilitó la legalización de formas "atípicas" de empleo, tales como flexibilidad horaria y trabajo temporario, así como también el cobro por productividad.

\section{2- ¿Qué sabemos sobre el teletrabajo y los teletrabajadores en Argentina? "Juntando o antes, o agora e o depois"4}

Si bien la definición de teletrabajo presenta una serie de aristas que son materia de debate, en términos generales es posible señalar que esta modalidad refiere a la realización de actos, ejecución o prestación de servicios en un lugar -o lugaresdiferente al establecimiento del empleador, mediante la utilización de tecnologías de la información y la comunicación. Más allá de las dificultades para circunscribir el concepto, lo cierto es que esta modalidad de trabajo posibilitó atenuar algunos de los efectos de las medidas implementadas para contener la pandemia de Covid-19, y permitió el funcionamiento de instituciones y empresas.

En Argentina, disponemos de escasa información sobre el teletrabajo, y esa información es anterior a la pandemia. Los datos disponibles provienen de la Encuesta de Indicadores Laborales (EIL) ${ }^{5}$, que, en el año 2017, incorporó un módulo sobre teletrabajo, y de la Encuesta Nacional a Trabajadores sobre Condiciones de 
Empleo, Trabajo, Salud y Seguridad (ECETSS) ${ }^{6}$, realizada a fines de 2018. Ambas encuestas fueron llevadas adelante por lo que hoy es el Ministerio de Trabajo, Empleo y Seguridad Social de la Nación. ${ }^{7}$ Los datos que se deprenden de estas encuestas son los siguientes:

- $\quad$ El teletrabajo era una modalidad incipiente en Argentina antes de la pandemia de Covid-19. Representaba entre un $8 \%$ (EIL) y un $10 \%$ (ECETSS) de los trabajadores.

- $\quad$ En solo el 3\% de las empresas privadas relevadas por la EIL hay personas que realizan teletrabajo.

- Los trabajadores que realizan teletrabajo suelen pertenecer a grandes empresas. Más de la mitad de las personas que teletrabajan lo hacen en empresas de más de 40 trabajadores (ECETSS).

- $\quad$ Entre las personas que teletrabajan, los varones superan a las mujeres.

- $\quad$ El nivel educativo de quienes realizan teletrabajo es considerablemente superior a la media: más de la mitad tiene estudios universitarios. Esto se relaciona con el hecho que casi el 15\% de los que realizan teletrabajo ocupan puestos directivos, de gestión y/o gestión financiera (ECETSS).

- Quienes realizan teletrabajo perciben ingresos superiores a la media (ECETSS).

- Los resultados de las encuestas no son coincidentes respecto de la información sobre los grupos etarios. De la ECETSS se desprende que no se observan grandes diferencias entre quienes realizan teletrabajo del resto de los trabajadores, mientras que de los datos de la EIL muestran que el 97,4\% de los teletrabajadores encuestados tienen entre 26 y 35 años.

- $\quad$ Si bien la mayoría de los teletrabajadores están registrados, existe un número no menor de trabajadores no registrados dentro de los teletrabajadores, que alcanza al 23\% (ECETSS).

- Respecto de la frecuencia semanal de trabajo bajo la modalidad de teletrabajo, los resultados de la EIL muestran que el 87,6\% de los teletrabajadores lo hace al menos un día por semana (medio día fue incluido en este rango). El restante $12,4 \%$ se distribuye en las categorías de 2 a 5 días de teletrabajo por semana. Por su parte, de la ECETSS se desprenden datos diferentes: $44 \%$ de los teletrabajadores lo hacen de manera exclusiva y $40 \%$ lo hacen un día o dos por semana.

Los analistas coinciden en que el escenario pospandemia seguramente será diferente, más cercano a una situación intermedia, donde no se volverá a la situación previa ni se permanecerá en altísimos niveles de teletrabajo.

Desde la perspectiva de Weller (2020), la proporción de trabajo susceptible a realizarse a distancia varía entre países por cuatro razones: 1) por las características de las ocupaciones, muchas de las cuales requieren presencia personal en el lugar de trabajo; 2) por las características de la estructura productiva de los países; 3) por la existencia de un gran sector informal donde el teletrabajo no es una opción real; y 4) porque la posibilidad del teletrabajo depende del nivel de infraestructura tecnológica, así como la posibilidad de acceder a ella, y la proporción de trabaja- 
dores con las competencias digitales necesarias. En esta dirección, y tomando en cuenta este último factor limitante, Albrieu (2020) calcula para nuestro país que la proporción de empleos para los cuales existiría la factibilidad tecnológica del teletrabajo, estimada en $27-29 \%$, baja a un $18 \%$ si se excluye a los ocupados que en sus hogares no cuentan con la infraestructura digital requerida para estos fines.

\section{3- La regulación sobre el teletrabajo: tres aspectos centrales}

La promulgación -el viernes 14 de agosto de 2020- de la Ley 27.555 relativa al régimen legal de contrato de teletrabajo forma parte del componente vinculado con la legislación laboral dentro de las denominadas "regulaciones directas del mercado de trabajo"8 (Cortés y Marshall, 1993; Marshall, 2000). La nueva regulación solo alcanza a quienes teletrabajan en el marco de las relaciones laborales previstas en la Ley de Contrato de Trabajo.

La regulación del trabajo y el empleo constituye una cuestión social clave que permanentemente reactualiza las prácticas y el debate sobre la correlación de fuerzas entre el capital y el trabajo. Dentro de toda institucionalización laboral, la regulación del tiempo de trabajo se erige en uno de los andamiajes centrales en torno al cual se articula buena parte de la normativa. En esa dirección, es fundamental señalar que la delimitación temporal entre la vida pública y privada constituyó parte del compromiso social creado en la esfera política entre el capital y el trabajo (Rubery et al., 2005). Este compromiso se consolida en el marco de la relación salarial fordista, y actúa regulando la extensión de jornada de trabajo de forma tal de establecer límites a la apropiación del tiempo de los trabajadores por parte de los empresarios. Conjuntamente, la separación entre un tiempo para la reproducción económica y para la reproducción social, así como la delimitación de la jornada, actuaron "protegiendo" el tiempo de la reproducción social, permitieron la sincronización de los tiempos - tanto hacia el interior de la fábrica como fuera de ella- y el establecimiento de los ritmos colectivos (Dedecca, 2004; Lallement, 2007).

La modalidad del teletrabajo conlleva la particularidad de poner en discusión algunas de las características centrales del modelo temporal surgido con la sociedad industrial, Presenta, así, una serie de desafíos para la nueva regulación. En este sentido, es necesario señalar dos elementos centrales. En primer lugar, el teletrabajo rompe la clásica unidad de tiempo y espacio que ha caracterizado al trabajo y deja de estar situado en un tiempo y en un lugar bien definidos y predeterminados (Méda, 2016). En segundo lugar, esta modalidad conlleva la difuminación de las fronteras entre el mundo de la vida y el mundo del trabajo, lo cual produce transformaciones en las formas de vivenciar las clásicas dicotomías entre tiempo de la producción/otros tiempos sociales, espacio laboral/espacio doméstico, trabajo/no trabajo. Las actividades laborales pasan a invadir crecientemente el día y la extensión de la jornada; la disponibilidad laboral y la intensificación de los ritmos de trabajo se convierten en rasgos típicos de esta modalidad de trabajo.

Tal como señalamos, por las propias características de esta modalidad aunadas a las transformaciones más generales en las formas de organización del trabajo, el teletrabajo impone una serie de desafíos a su regulación. En esta dirección, abor- 
daremos tres elementos que introduce la nueva institucionalidad vinculados con la regulación del tiempo de trabajo.

Los primeros dos elementos solo pueden analizarse en forma conjunta y refieren al establecimiento de la jornada, ya sea en lo convenido por hora o por objetivo, y al derecho a la desconexión digital por las características propias de la modalidad objeto de regulación. Aunque difíciles de implementar, ambas cuestiones constituyen aspectos centrales a ser regulados, ya que la evidencia internacional muestra altos niveles de horas extra realizadas y no pagas dentro de la modalidad de teletrabajo. En este sentido, el derecho a la desconexión digital tiene por objetivo limitar el trabajo dentro de los horarios y en la cantidad de horas acordadas. A la vez, intenta restablecer las fronteras y los límites entre el mundo de la vida y el mundo del trabajo. De esta forma, actúa constituyendo una protección legal ante la disponibilidad temporal o "teledisponibilidad" asociada a esta modalidad. La desconexión se plantea en una doble dirección: actúa como un derecho hacia los trabajadores en tanto el empleador no podrá exigir la realización de tareas fuera de la jornada laboral y actúa, también, como una obligación para el empleador, quien no podrá remitir comunicaciones fuera de los horarios pautados. De esta forma, la delimitación de la jornada de trabajo actúa como un mecanismo para mejorar la calidad de vida de las personas y se enlaza con los aportes de los enfoques y del movimiento feminista, que colocan en un nuevo plano la importancia de las actividades laborales no mercantiles.

Así, la Ley 27.555 incorpora por primera vez en la legislación laboral de nuestro país las tareas de cuidado como derecho para quienes realizan el trabajo desde el hogar. Las tareas de cuidado que la Ley contempla son aquellas dirigidas a personas menores de trece años, personas con discapacidad o adultas mayores que convivan con la persona trabajadora y que requieran de asistencia específica. El articulado excluye, de esta forma, la responsabilidad de los cuidados fuera del hogar.

La centralidad de esta innovación en la legislación laboral se encuentra en llevar al espacio de lo público -y, por lo tanto, al espacio de lo político- el conflicto entre las actividades y el tiempo que implica la articulación entre la vida laboral y la vida familiar. Esta articulación conflictiva deja de constituir una cuestión social secundaria y, en definitiva, del mundo de lo privado. La nueva norma del tiempo de trabajo se modifica para hacer posibles los "cuidados" (Prieto, 2007). Argentina incorpora -40 años después- algunas de las recomendaciones presentes en la CE$\mathrm{DAW}^{9}$, con lo cual da un primer paso en la jerarquización del trabajo de cuidados y lo contempla de manera equitativa, sin distinción de géneros.

Si bien el texto de la norma no incorpora la noción de "conciliación", es la idea que está presente, de forma clara. Esta noción se cristaliza en las décadas de 1980 y 1990 en los documentos de los organismos internacionales que generaban recomendaciones a los Estados para el diseño e implementación de políticas públicas tendientes a la equiparación del acceso de varones y mujeres al mercado de trabajo. De esta forma, surgen las denominadas "políticas de conciliación" entre el ámbito laboral y familiar como respuesta al problema (Delfino, Pagura y Logiodice, 2019). 
Desde inicio del siglo XXI, la noción de "conciliación" y las políticas que se derivaron de esas recomendaciones fueron puestas en discusión en la medida en que constituyeron verdaderas políticas de empleo diseñadas desde una lógica capitalista-mercantil y sirvieron exclusivamente para que quienes "concilien" sean las mujeres, en un intento de armonizar la inserción laboral con las tareas de cuidado dentro de un esquema que no ponía en discusión la división sexual del trabajo.

Este es el gran marco que introduce la nueva Ley, en el cual se delega la responsabilidad de la reglamentación de sus especificidades, sobre todo sectoriales, y su aplicación en las negociaciones colectivas de trabajo. Esto deriva en dos cuestiones centrales que deberán estar presentes en esas negociaciones para que la jerarquización del trabajo de cuidado no se convierta en una cuestión meramente declamativa. El primer elemento que las negociaciones colectivas deben contemplar son los mecanismos para facilitar que las actividades de cuidado no vuelvan a recaer casi exclusivamente sobre las mujeres. Esto solo es posible mediante la promoción de la corresponsabilidad en relación con el trabajo doméstico y de cuidados no remunerado, y posibilitando que no quede circunscripto a las posibles resoluciones que tengan las familias en el ámbito de los hogares. Allí se presenta la segunda cuestión que debe estar considerada: los servicios de cuidado. Es claro que la necesidad de servicios públicos de cuidados de calidad es un elemento indispensable, pero el modelo debería contener otros instrumentos pasibles de ser introducidos en las negociaciones colectivas, tales como liberación de tiempo para tareas de cuidado - licencias con y sin goce de salario- y oferta de servicios de cuidados como guarderías, cuidados para adultos mayores y personas con discapacidades, entre otros.

\section{A manera de cierre}

En términos de tiempo de trabajo, la nueva regulación introduce algunos aspectos interesantes e innovadores en la legislación laboral de nuestro país, incluso saldando deudas que llevaban varias décadas. Paradójicamente, algunos de estos elementos introducidos hoy presentan aristas más complejas que la Ley ha soslayado.

El hecho que la Ley deje un amplio margen de debate, reglamentación y aplicación a las negociaciones colectivas nos permite preguntarnos: ¿podrán las negociaciones colectivas ser superadoras de la Ley marco? Es claro que hoy no tenemos respuesta a esta pregunta, pero el futuro no parece ser muy auspicioso por varias razones. En los últimos años, en los cuales la coalición gobernante no logró su objetivo de sancionar una reforma laboral integral, fueron los convenios colectivos los instrumentos a través de los cuales se introdujeron reformas que profundizaron la flexibilización laboral. Por otra parte, las demandas de igualdad entre los géneros para que se incluyan políticas vinculadas al trabajo de cuidados no han permeado, o lo han hecho de forma muy parcial y débil, la agenda sindical. Estas dos cuestiones se enlazan con una tercera, relativa a que la participación femenina hacia el interior de los espacios sindicales continúa siendo escasa, y lo mismo acontece con la democratización de las posiciones jerárquicas en estas organizaciones. 
¿Podrá un sindicalismo que no se renueve incorporar a su agenda la defensa de problemáticas clásicas como la regulación de la jornada de trabajo, junto con la introducción de una perspectiva de género como forma de reglamentar la Ley de teletrabajo?

\section{Referencias}

1. DNU No 297/20 del 19 de marzo de 2020.

2. Resolución MTEySS No 202/20 del 13 de marzo de 2020 (para personas confirmadas de COVID-19, casos sospechosos, "contactos estrechos", procedentes de zonas afectadas); Resolución MTEySS N 207/20 del 16 de marzo de 2020 (para mayores de 60 años, embarazadas y grupos de riesgo); y Resolución MTEySS N 279/20 del 30 de marzo de 2020 (estipula que los trabajadores alcanzados por dicho ASPO quedaban dispensados del deber de asistencia).

3. La Ley no tiene vigencia inmediata y comenzará a regir en los 90 días posteriores a la finalización del período de emergencia.

4. Refiere a un verso de la letra de "Sozinho", del músico y poeta brasileño Caetano Veloso.

5. La EIL es un programa estadístico existente desde 1998. En la actualidad, la encuesta releva en doce centros urbanos a empresas privadas formales a partir de 5 y 10 trabajadores (según aglomerado), pertenecientes a todas las ramas de actividad, con excepción del sector de actividades primarias (agropecuario y minas y canteras).

6. La ECETSS es realizada a trabajadores ocupados de 15 años o más, en localidades de más de 2000 habitantes pertenecientes a las 23 provincias y a la Ciudad Autónoma de Buenos Aires (asalariados en unidades productivas registradas y no registradas, trabajadores por cuenta propia, patrones y trabajadores en casas particulares).

7. Es fundamental tener en cuenta que, dadas las características de una y otra encuesta, sus datos no son comparables.

8. El segundo componente de las políticas laborales son las medidas hacia el mercado de trabajo.

9. La Convención sobre la Eliminación de toda forma de Discriminación contra la Mujer (CEDAW, por sus siglas en inglés) fue aprobada por Naciones Unidas en 1979.

\section{Bibliografía}

Adam, B. (1995). Timewatch. The Social Analysis of Time. Cambridge, USA: Polity Press

Albrieu, R. (2020). Evaluando las oportunidades y los límites del teletrabajo en Argentina en tiempos del COVID-19. CIPPEC.

Bouffartigue, P. (2012). La disponibilité temporelle au travail: nouvelles formes, nouveaux enjeux. Temps de travail et travail des temps, Oct., Strasbourg, France.

Bouffartigue, P. y Boutellier, J. (2002). L'érosion de la norme du temps de travail. Travail et Emploi, (2), Octobre, 43-55.

Cortés, R. y Marshall, A. (1993). Modelo de crecimiento, intervención social del Estado y regulación de la fuerza de trabajo. Argentina, 1890-1990. En Reestructuración y regulación institucional del mercado de trabajo. Ginebra, Suiza: OIT.

Dedecca, C. S. (2004). Tempo, trabalho e gênero. En A. Costa, E. Menicucci de Oliveira, M. E. Bezerra de Lima y V. Soares. (Org.), Reconfiguração das relações de gênero no trabalho, pp. 21-52. São Paulo, Brasil: CUT.

Delfino, A., Pagura. F. y Logiodise, L. (2019). ¿“Conciliación” o “articulación” entre trabajo remunerado y trabajo doméstico y de cuidados no remunerado? Genealogía de una discusión. En XIV Jornadas nacionales de Historia de las Mujeres y IX Congreso Iberoamericano de Estudios de Género. Mar del Plata, Argentina, julio-agosto.

Elias, N. (1998). Sobre o tempo. Rio de Janeiro, Brasil: Jorge Zahar Editores.

Giddens, A. (1991). As conseqüencias da modernidade. São Paulo, Brasil: Editora UNESP.

Lallement, M. (2007). Tiempo, trabajo, sujeto. Balance, cuestiones clave y perspectivas de las trans- 
formaciones contemporáneas. En C. Prieto (Ed.), Trabajo, género y tiempo social, pp. 49-63. Madrid, España: Editorial Hacer - Editorial Complutense.

Marshall, A. (2000). Regulación del mercado de trabajo, salarios y disciplina laboral. Un análisis comparativo. En Congreso Latinoamericano de Sociología del Trabajo. ALAST, Buenos Aires, Argentina.

Méda, D. (2016). The Future of Work: The Meaning and Value of Work in Europe. ILO Research Paper No. 18. Geneva, Italy: International Labour Office.

Prieto, C. (2007). De la "perfecta casada" a la "conciliación de la vida familiar y laboral". En C. Prieto (Ed.). Trabajo, género y tiempo social, pp. 21-48. Madrid, España: Editorial Complutense.

Prost. A. (1989). Fronteras y Espacios de lo Privado. En P. Ariès y G. Duby (Dirs.), Historia de la Vida Privada. Buenos Aires, Argentina: Taurus.

Rubery, J., Ward, K., Grimshaw, D., y Beynon, H. (2005). Working Time, Industrial Relations and the Employment Relationship. Time and Society, 14 (1), London, UK: Sage.

Thompson, E. P. (1984). Tiempo, disciplina de trabajo y capital industrial. En, E. P. Thompson, Tradición, revuelta y conciencia de clase. Estudios sobre la crisis de la sociedad preindustrial, pp. 239-293. Barcelona, España: Crítica.

Weller, J. (2020). La pandemia del COVID-19 y su efecto en las tendencias de los mercados laborales. Documentos de Proyectos 45759. Naciones Unidas, Comisión Económica para América Latina y el Caribe (CEPAL).

Andrea Delfino, "La irrupción del teletrabajo y el eterno retorno al debate sobre la regulación del tiempo de trabajo". Revista Temas y Debates. ISSN 1666-0714, año 24, número especial, julio-diciembre 2020, pp. 215-224. 